\title{
A Novel Algorithm for Distributed Dynamic Interference Coordination in Cellular Networks
}

\author{
Marc C. Necker \\ Institute of Communication Networks and Computer Engineering, University of \\ Stuttgart, Pfaffenwaldring 47, Germany, *
}

\begin{abstract}
Wireless systems based on Orthogonal Frequency Division Multiple Access (OFDMA) multiplex different users in time and frequency. One of the main problems in OFDMA-systems is the inter-cell interference. A promising approach to solve this problem is interference coordination (IFCO). In this paper, we present a novel distributed IFCO scheme, where a central coordinator communicates coordination information in regular time intervals. This information is the basis for a local inner optimization in every basestation. The proposed scheme achieves an increase of more than $100 \%$ with respect to the cell edge throughput, and a gain of about $30 \%$ in the aggregate spectral efficiency compared to a reuse 3 system.
\end{abstract}

Keywords and phrases 3GPP LTE, 802.16e, OFDMA, WiMAX, beamforming, graph coloring, interference coordination, scheduling

Digital Object Identifier 10.4230/OASIcs.KiVS.2011.233

\section{Introduction}

IFCO has been an active topic especially in the $3 \mathrm{GPP}$ standardization body. It is a promising approach to solve the problem of inter-cellular interference in a reuse 1 scenario. Most activities have focused on local schemes operating on local state information in every basestation. These schemes are often based on power regulation [4] or Fractional Frequency Reuse (FFR) [3]. A number of FFR-based schemes was compared in [14] and [13]. Another local scheme was proposed by Xiao et al. in [15]. Kiani et al. propose a distributed scheme based on local measurements [7]. The authors measure the increase in the overall network capacity, but do not consider fairness issues, such as the throughput at the cell edge. In [8], Li et al. propose a distributed scheme by formulating a local and a global optimization problem, thus being able to include global state information in the coordination process. They consider one strongest interferer and do not consider fairness issues. However, fairness is crucial since it is easy to sacrifice cell edge throughput in favor of overall network throughput [12].

In this paper, we present a novel distributed interference coordination scheme. We explicitly consider the performance at the cell edge compared to the aggregate throughput as a fairness metric. A central coordinator solves an outer optimization problem based on global information collected from the basestations, and the basestations solve a local inner optimization problem based on local state information. The communication with the central coordinator can be in intervals in the order of seconds. The performance is significantly increased compared to Coordinated FFR [10] and pushed further towards the (theoretical) performance of a globally coordinated system. In particular, the presented scheme outperforms a frequency reuse 3 sytem by more than $100 \%$ with respect to the cell edge throughput while increasing the aggregate throughput performance by more than $30 \%$.

\footnotetext{
* Work was done at the University of Stuttgart. The author thanks Prof. Kühn and Prof. Tran-Gia for their great support and many invaluable discussions. He is now with Daimler AG, marc.necker@daimler.com. 


\section{Overview of Cellular 802.16e System Model}

We consider a cellular 802.16e-system [6], focusing on the Adaptive Modulation and Coding (AMC) zone in the downlink subframe. We consider the AMC 2x3 mode, which defines subchannels of 16 data subcarriers by 3 OFDM-symbols. Our scenario consists of a hexagonal cell layout comprising 19 basestations at a distance of $d_{\mathrm{BS}}=1400 \mathrm{~m}$ with $120^{\circ}$ cell sectors. The scenario is simulated with wrap-around, making all cells equal with no distinct center cell. All cells were assumed to be synchronized on a frame level. Every basestation has 3 transceivers, each serving one cell sector. The transceivers are equipped with linear array beamforming antennas with 4 elements and gain patterns according to [9].

\section{Graph-Based Interference Coordination}

In [9], we introduced a scheme for global interference coordination based on an interference graph. In this graph, the vertices represent the mobile terminals, and the edges represent critical interference relations between them. It is constructed by evaluating the interference that a transmission to one mobile terminal causes to any other terminal. For each terminal, we first calculate the total interference that the mobile receives from other basestations within a radius of $d_{i c}$. We then block the largest interferers from using the same set of resources by establishing a relation in the interference graph. This is done such that a desired minimum SIR $D_{S}$ is achieved. Note that if $d_{i c}=0$, only interference from other sectors of the same basestation is considered, whereas if $d_{i c} \geq 1$ inter-cellular interference from other basestations is considered as well. For more details, please refer to [12].

Resources need to be assigned to the mobile terminals such that no two mobile terminals are assigned the same resources if they are connected in the graph. This is equal to coloring the graph if the resources correspond to colors [12]. Resources may be mapped to colors $c_{k, i} \in \mathbf{C}$ as shown in Fig. 1. Every AMC zone is subdivided into a certain number $N_{p}$ of resource partitions. Several AMC zones in subsequent MAC frames form one virtual frame such that the total number of resource partitions in the virtual frame corresponds to the number of required colors during the coloring process (precisely: to the next larger multiple of $N_{p}$ ).

\section{Distributed Interference Coordination}

In this section, we present a novel scheme for distributed IFCO. The scheme uses a central coordinator which is responsible for the coordination of neighboring basestations based on a global interference graph with $d_{i c} \geq 0$. At the same time, every basestation creates a local interference graph with $d_{i c}=0$ to coordinate the transmissions in its three sectors. The scheme takes a formal approach by formulating an inner optimization problem, which needs to be solved by every basestation. This inner optimization problem is subject to constraints delivered by the outer optimization problem, which is solved in the central coordinator.

\subsection{Outer Optimization Problem}

The outer optimization problem is solved by the central coordinator based on an interference graph with $d_{i c} \geq 1$. This graph creation may be based on measurements obtained from the mobile terminals and collected by the central coordinator which is out of the scope of this paper. The goal of the outer optimization problem is to find a set of colors $\mathbf{C}_{i} \subseteq \mathbf{C}$ (i.e., a set of resource partitions) for every mobile terminal $m_{i}$ such that there is no conflict between any combination of colors in the sets. This problem is known as fractional graph coloring. An 


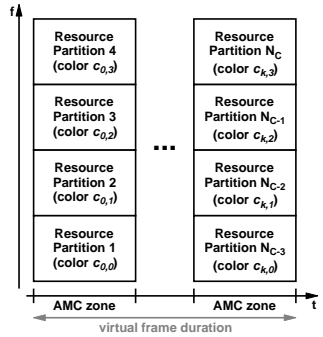

Figure 1 Resource partitioning with $N_{p}=4$

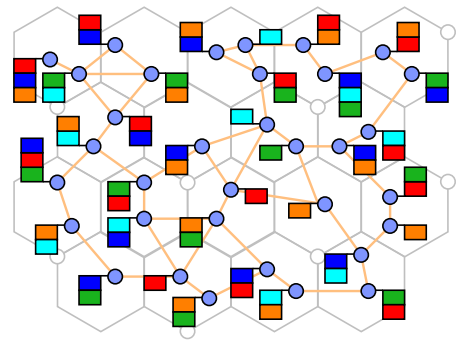

Figure 2 Graph coloring of outer optimization problem.

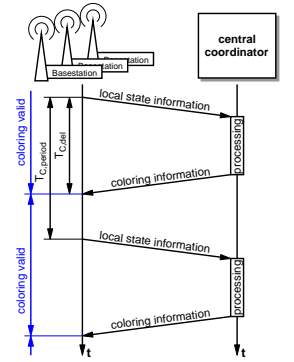

Figure 3 Timing diagram

example for a possible coloring is shown in Fig. 2. Fractional graph coloring is NP-hard. We take the following approach to solve the outer optimization problem. We first color the graph by means of the sub-optimal heuristic Dsatur [5]. Next, we traverse all mobile terminals in a random order and assign a second color to every mobile terminal where possible. We repeat this step until no more extra colors can be assigned to any mobile terminal.

\subsection{Inner Optimization Problem}

The goal of the inner optimization problem is to assign every mobile terminal to one or more resource partitions $c_{k, l}$ of the respective cell sector. This means that every mobile terminal is assigned a set of colors $\mathbf{R}_{i} \subseteq \mathbf{C}_{i}$ on which it is served. That is, $\mathbf{R}_{i}$ must be chosen from the color set $\mathbf{C}_{i}$ assigned to mobile terminal $m_{i}$ by the coordinator. To formulate the optimization problem we introduce for every mobile $m_{i}$ the matrix $\left(x_{i, k, l}\right)$, which describes the resource allocation for a particular cell sector:

$$
x_{i, k, l}=\left\{\begin{array}{ll}
1 & \text { if mobile } m_{i} \text { is served in resource } c_{k, l} \\
0 & \text { if mobile } m_{i} \text { is not served in resource } c_{k, l}
\end{array} .\right.
$$

This means that $\mathbf{R}_{i}$ can be defined as

$$
\mathbf{R}_{i}=\left\{c_{k, l} \mid x_{i, k, l}=1\right\} .
$$

We further define a utility $u_{i}$ for every mobile terminal $m_{i} \cdot u_{i}$ is a real number and denotes the utility if the mobile terminal is scheduled in a MAC frame.

The objective function of the inner optimization problem for basestation $b$ then is

$$
\max \left(\sum_{m_{i} \in M_{b}} \sum_{k} \sum_{l} u_{i} x_{i, k, l}\right),
$$

where $M_{b}$ contains all mobiles $m_{i}$ which are served by any of the three transceivers of basestation $b$. Note that eq. (3) maximizes the utility sum for one virtual frame. Hence, the resource allocation problem has to be solved at the beginning of every virtual frame.

The inner optimization problem is subject to a number of constraints.

1. Every mobile $m_{i}$ has to be served using one color from $\mathbf{C}_{i}$ assigned by the coordinator:

$$
\forall\left\{x_{i, k, l} \mid x_{i, k, l}=1\right\}: c_{k, l} \in \mathbf{C}_{i} .
$$

2. Every mobile terminal has to be served at least once in every virtual frame:

$$
\forall i: \sum_{k} \sum_{l} x_{i, k, l} \geq 1 \text {. }
$$

3. Every mobile terminal must not be served more than once per MAC frame:

$$
\forall i \forall k: \sum_{l} x_{i, k, l} \leq 1
$$


4. The constraints of the local interference graph have to be met:

$$
\forall\left\{(i, j) \mid e_{i j}=1\right\}: \mathbf{R}_{i} \cap \mathbf{R}_{j}=\varnothing
$$

The optimization problem formulated by equations (3)-(7) is a binary integer linear program (BILP), which is NP-hard. BILPs can be treated by standard optimization packages for integer linear programs (ILPs), but represent a particularly difficult class of ILPs.

\subsection{System Architecture}

The system architecture comprises a central coordinator which creates the interference graph and solves the outer optimization problem. All basestations communicate the necessary data to the central coordinator, as shown in Fig. 3. The set of colors is then communicated from the central coordinator to the basestations, which periodically solve the inner optimization problem. Communication with the central coordinator takes place with an update period $t_{C, u p}$. The delay $t_{C, \text { delay }}$ contains all signalling delays, processing delays and synchronization delays.

\section{Solution of Inner Optimization problem with Genetic Algorithms}

The inner optimization problem can be solved by means of genetic algorithms. Genetic algorithms are based on generations of solutions. Every generation contains a number $|P|$ of possible solutions to the optimization problem, which are called genomes. Every genome is evaluated and assigned a fitness value. This fitness value is usually a real number and indicates how "good" the solution is. For details about the applied genetic algorithm see [11].

The genetic representation of a solution is problem-specific and often not obvious. For our problem, we choose a list representation as detailed in [11]. The list contains references to the mobile terminals along with the colors $\mathbf{C}_{i}$ that were assigned during the outer optimization. The order of the mobile terminals in the list determines the assignment of resources to each mobile terminal. To assign resources, a placement algorithm traverses the list and assigns the first possible and free resource partition to the mobile terminals. The resource partitions must not yet be occupied, and the assignment must not be in conflict with the inner interference graph.

The placement algorithm takes care that constraints (4), (6), and (7) are fulfilled. Constraint (5) is taken into account during the subsequent evaluation of the genome by counting the number $n_{u}$ of mobile terminals that have not been assigned resources. The second factor during the evaluation is the number $n_{o}$ of occupied resource partitions (i.e., the resource utilization). Finally, we set $u_{i}=1$ for all mobiles $m_{i}$. Hence, the number of scheduled mobile terminals will be maximized, i.e., it will be attempted to schedule a mobile terminal in every resource partition. The overall fitness of a genome is then calculated as Fitness $=n_{o}-n_{u}$.

\section{Performance Evaluation}

\subsection{16e scenario and simulation model}

We consider a system with a bandwidth of $10 \mathrm{MHz}$ and a MAC-frame-length of $5 \mathrm{~ms}$. The AMC zone was set to 9 OFDM-symbols with $48 \cdot 3$ subchannels. AMC was applied from QPSK 1/2 to 64QAM 3/4. This results in a max. raw data rate of $6.2 \mathrm{Mbps}$ in the AMC zone.

The system model was implemented as a frame-level simulator using the event-driven simulation library IKR SimLib [1] with a detailed physical layer model as described in [11]. Throughput measurements were done on the IP-layer in downlink direction with greedy traffic sources, capturing all effects of SINR- and BLER-variations, retransmissions by ARQ and HARQ with chase combining, and MAC overhead. 


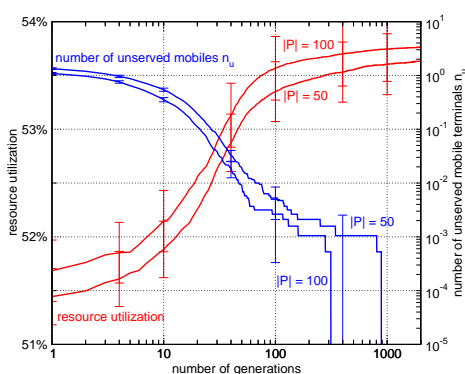

Figure 4 Monte-Carlo Runs: Convergence of algorithm

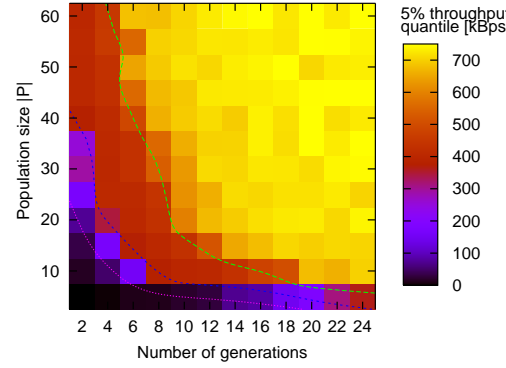

Figure 5 Monte-Carlo Runs: Tradeoff between complexity and performance

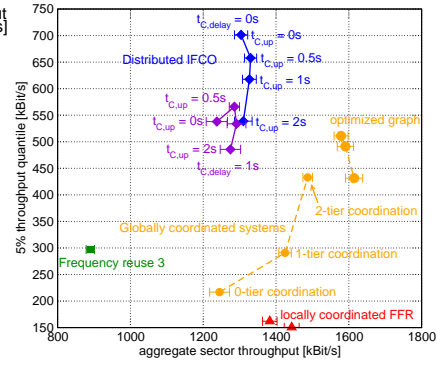

Figure 6 Mobile scenario: Compariosn with other IFCOschemes.

We consider two mobility scenarios. In the static scenario, Monte-Carlo-like simulations are performed, where $N=9$ terminals are randomly replaced for every drop. The drops have a duration of $4 \mathrm{~s}$ with full event-driven simulation. In the mobile scenario, each cell sector contains $N=9$ fully mobile terminals moving at a velocity of $30 \mathrm{~km} / \mathrm{h}$, which are restricted to their respective cell sector (see [9]). The Monte-Carlo runs were used to explore the parameter space, since they are much faster to perform, whereas a fully time-continuous simulation was performed in the mobile scenario to achieve final performance values.

The considered throughput performance metrics are the aggregate system throughput, which is proportional to the overall spectral efficiency, and the $5 \%$ quantile of the individual throughputs of all terminals, which correlates with the throughput of terminals close to the cell edge [2]. Hence, the $5 \%$ quantile is a very good fairness indicator.

\subsection{Convergence and Complexity of genetic algorithm}

The convergence behavior is shown in Fig. 4. Plotted is the overall resource utilization and the average number of unserved terminals after a certain number of generations $N_{\text {gen }}$. A higher resource utilization leads to a larger aggregate throughput, while the number of unserved terminals affects the fairness. In particular, terminals in unfavorable positions at the cell edge will most likely be unserved for small $N_{g e n}$, thus decreasing the cell edge performance. From Fig. 4 we can see that as few as $N_{\text {gen }}=10$ generations bring the number of unserved terminals below one. For $N_{\text {gen }}=100$, the algorithm already comes close to its optimum performance. The graph also shows that the aggregate throughput, which is mainly determined by the resource utilization, depends much less on the number of generations than the cell edge throughput. This also holds for the population size $|P|$.

The computational complexity is mainly proportional to $N_{g e n} \cdot|P|$. Figure 5 plots the $5 \%$ throughput quantile depending on $N_{g e n}$ and $|P|$. The chart shows that the best performance for a particular computational effort can be achieved for $|P| \approx 2 N_{\text {gen }}$. Figure 5 further shows that it requires only a small computational effort to achieve near optimal results.

\subsection{Comparison with existing IFCO schemes}

Figure 6 compares the performance of the proposed distributed coordination scheme with an uncoordinated frequency reuse 3 system (also with beamforming antennas), and a system with FFR, which is locally coordinated based on local state information in every basestation (see [13]). Furthermore, the chart contains reference curves with global coordination according to [9] and [10]. All results in Fig. 6 were obtained in the mobile scenario with only $N_{g e n}=20$.

The performance of the proposed distributed IFCO scheme is plotted for different values

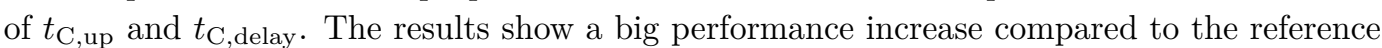
frequency reuse 3 system, and the cell edge performance compared to a locally coordinated 
FFR system is greatly increased. Even for update periods and delays in the order of seconds the IFCO schemes achieves a large performance gain. Note also that in a nomadic scenario, which is a realistic use case for $802.16 \mathrm{e}$ networks, the performance gain will be mostly independent of $t_{\mathrm{C} \text {,up }}$ and $t_{\mathrm{C} \text {,delay }}$, hence the performance gains will even be larger compared to the mobile scenario when $t_{\mathrm{C} \text {,up }}$ and $t_{\mathrm{C} \text {,delay }}$ are in the order of seconds.

\section{Conclusion}

We efficiently solved the problem of IFCO by separating the initial global optimization problem into two separate problems, namely the inner and the outer optimization problem. We presented efficient approaches to solve these problems based on graph coloring heuristics and genetic algorithms. The complexity is well manageable, since the genetic algorithm converges after very few generations, allowing for efficient hardware-based real-time implementations. We evaluated the performance in a fully mobile scenario. The proposed scheme outperforms a reference reuse 3 system by more than $30 \%$ with respect to the aggregate spectral efficiency, and by more than $100 \%$ with respect to the cell edge throughput.

\section{References}

1 IKR Simulation Library. [Online] http://www.ikr.uni-stuttgart.de/Content/IKRSimLib/.

2 3GPP TS25.814. Physical layer aspects for evolved Universal Terrestrial Radio Access (UTRA) (Rel. 7). 3rd Gen. Partnership Project, June 2006.

3 3GPP TSG RAN WG1\#42 R1-050841. Further analysis of soft frequency reuse scheme. Technical report, 3rd Gen. Partnership Project, 2005.

4 3GPP TSG RAN WG1\#47bis R1-070040. DL power allocation for dynamic interference avoidance in E-UTRA. Technical report, 3GPP, Sorrento, Italy, January 2007.

5 D. Brélaz. New methods to color the vertices of a graph. Comm.ACM, 22(4):251-256, 1979.

6 IEEE 802.16e. IEEE Std. for Local and metropolitan area netw., Part 16: Air Interface for Fixed and Mobile Broadband Wireless Access Systems, Amendment 2: Phys. and Medium Access Control Layers for Combined Fixed and Mobile Op. in Licensed Bands, Feb. 2006.

7 Saad G. Kiani, Geir E. Oien, and David Gesbert. Maximizing multicell capacity using distributed power allocation and scheduling. In Proc. IEEE WCNC 200\%, Kowloon, China.

8 Guoqing Li and Hui Liu. Downlink dynamic resource allocation for multi-cell OFDMA system. In Proc. IEEE VTC 2003-Fall, Orlando, FL, USA.

9 M. C. Necker. Towards frequency reuse 1 cellular FDM/TDM systems. In Proc. ACM/IEEE MSWiM 2006, pages 338-346, Torremolinos, Spain, Oct. 2006.

10 M. C. Necker. Coordinated fractional frequency reuse. In Proc. ACM/IEEE MSWiM 200\%, Chania, Crete Island, October 2007.

11 M. C. Necker. A graph-based scheme for distributed interference coordination in cellular OFDMA networks. In Proc. 67th IEEE VTC2008 - Spring, Singapore, May 2008.

12 Marc C. Necker. Integrated scheduling and interference coordination in cellular OFDMA networks. In Proc. Broadnets, Raleigh, NC, Sep. 2007.

13 Marc C. Necker. Local interference coordination in cellular 802.16e networks. In Proc. IEEE VTC 2007-Fall, Baltimore, MA, Oct. 2007.

14 Arne Simonsson. Frequency reuse and intercell interference co-ordination in E-UTRA. In Proc. IEEE VTC 2007-Spring, pages 3091-3095, Dublin, Ireland, April 2007.

15 Weimin Xiao, R. Ratasuk, A. Ghosh, R. Love, Yakun Sun, and R. Nory. Uplink power control, interference coordination and resource allocation for 3GPP E-UTRA. In in Proc. IEEE VTC-2006, pages 1-5, 2006. 